"This accepted author manuscript is copyrighted and published by Elsevier. It is posted here by agreement between Elsevier and MTA. The definitive version of the text was subsequently published in [APPLIED SURFACE SCIENCE 314 (2014) 367-375, 10.1016/j.apsusc.2014.07.009]. Available under license CC-BYNC-ND."

\title{
Surface modification of polymeric materials by cold atmosphericplasma jet
}

\author{
K.G. Kostov ${ }^{\text {a,* }}$, T.M.C. Nishime ${ }^{\text {a }}$, A.H.R. Castro ${ }^{\text {a }}$, A. Toth ${ }^{b}$, L.R.O. Hein ${ }^{\text {a }}$ \\ ${ }^{a}$ Faculty of Engineering in Guaratinguetá-FEG, Universidade Estadual Paulista-UNESP Guaratiguetá, 12516-410, SP, Brazil \\ ${ }^{\mathrm{b}}$ Institute of Material and Environmental Chemistry, Hungarian Academy of Science P.O. Box 17, H-1525, Budapest, Hungary
}

\begin{abstract}
In this work we report the surface modification of different engineering polymers, such as, polyethylene terephthalate (PET), polyethylene (PE) and polypropylene (PP) by an atmospheric pressure plasma jet (APPJ). It was operated with Ar gas using $10 \mathrm{kV}, 37 \mathrm{kHz}$, sine wave as an excitation source. The aim of this study is to determine the optimal treatment conditions and also to compare the polymer surface modification induced by plasma jet with the one obtained by another atmospheric pressure plasma source the dielectric barrier discharge (DBD). The samples were exposed to the plasma jet effluent using a scanning procedure, which allowed achieving a uniform surface modification. The wettability assessments of all polymers reveal that the treatment leads to reduction of more than $40^{\circ}$ in the water contact angle (WCA). Changes in surface composition and chemical bonding were analyzed by $\mathrm{x}$-ray photoelectron spectroscopy (XPS) and Fourier-Transformed Infrared spectroscopy (FTIR) that both detected incorporation of oxygen-related functional groups. Surface morphology of polymer samples was investigated by Atomic Force Microscopy (AFM) and an increase of polymer roughness after the APPJ treatment was found. The plasma-treated polymers exhibited hydrophobic recovery expressed in reduction of the O-content of the surface upon rinsing with water. This process was caused by the dissolution of low molecular weight oxidized materials (LMWOMs) formed on the surface as a result of the plasma exposure.
\end{abstract}

\section{Introduction}

Non-equilibrium atmospheric pressure plasmas [1-4] have attracted considerable attention because they require low capital cost, can be easily implemented in a continuous production line and also due to their ability to generate highly reactive chemical species at ambient gas temperature [5]. Plasma jets, or plasma plumes are a kind of atmospheric pressure gas discharges where the plasma (usually obtained from a noble gas) is extended beyond the plasma generation region into the surrounding ambience $[4,6,7]$. The plasma plume length can extend up to several centimeters and can be adjusted by an electric field, a gas flow or a pressure gradient $[8,9]$. The interaction between the plasma plume and the surrounding atmosphere gives rise to different reactive species that may not be present in the discharge region $[10,11]$. The gas temperature of plasma jets was estimated by various diagnostic methods $[12,13]$ showing that if jet operation conditions were properly

\footnotetext{
* Corresponding author. Tel.: +55 12 31232180; fax: +55 1231232840 .

E-mail addresses: kostov@feg.unesp.br, konstantin_kostov2002@yahoo.com (K.G. Kostov).
}

chosen the gas temperature at the tip of the plasma plume could be quite low (around room temperature). The plasma jets are particularly useful for material treatment, since the gas flow from the jet guides the reactive species close to the surface where they cause surface etching and functionalization [14,15]. Another advantage of the atmospheric pressure plasma jets (APPJs) is that they are not confined by physical walls, thereby making the treatment of irregular-shaped 3D objects possible [16]. However, one limiting factor is the size of the treatment area, which in most APPJ treatments is around $1 \mathrm{~cm}^{2}$ [17]. To overcome this shortcoming arrays of many individual plasma jets have been developed [18,19]. Another manner for achieving a uniform surface modification is the manipulation of the jet and/or the sample [20,21]. On the other hand, a localized treatment extended over a limited area is desirable for applications in medicine, where for example, only cancer cells have to be targeted without destroying the surrounding healthy tissue. Also, plasma jets in the micrometer scale can be a very useful tool for surface engineering in different fields such as light emission in display panels, lithography, micro and nanotechnology [22-24].

However, still there exist great challenges in understanding the plasma jet physics and chemistry that impede the widespread application of APPJs as a surface modification tool. First of all, jet 
dynamic is not quite understood yet because besides the electromagnetic interaction it also involves the gas flow dynamics, thermal instabilities etc. Also, many different plasma jet configurations with power sources operating over a very large frequency range $(1 \mathrm{kHz}-50 \mathrm{MHz})$ are reported in the literature [25-29]. The most common characteristics of plasma jets are summarized in a recent review paper [4].

Operating the jet in ambient air implies collisions, recombination and plasma chemical reactions with the surrounding atmosphere. Therefore, the distribution of the active species in the discharge region, in the effluent, and close to the target may be significantly different [30-32]. For applying the APPJ as a material-processing tool, it is essential to understand the interaction between the active species in the jet downstream point with the surface and determine the size of the modified region as a function of jet parameters [33]. In this work we investigate the surface modification of different polymers by an Ar plasma jet at different operation conditions to find out the optimal process parameters. Comparison between the polymer surface modifications induced by a plasma jet and a DBD treatment has been conducted and the conclusion is that the APPJ is an efficient tool for surface modification. Of course, for obtaining good results the treatment parameters and the plasma jet operation conditions have to be optimized.

\section{Experimental setup and diagnostics methods}

\subsection{Materials}

Prior the routine surface modification of polymers a preliminary plasma jet treatment was conducted. It was aimed to evaluate the size and the location of the plasma-modified spot on the polymer surface as a function of plasma jet parameters and process conditions. This investigation required a large number of samples that had to be cheap and easy to handle. For this purpose we selected a commercial PET (0.3-mm-thickness) cut from colorless plastic bottles. The results from this initial study were used to establish a procedure for the following APPJ treatments that can provide a uniform surface modification over a selected area. To evaluate the surface chemical modification, induced by the APPJ treatment, two typical engineering polymers - polyethylene (PE) and polypropylene (PP), with a simple structure and a high chemical purity, were used. They were provided in the form of $0.1-\mathrm{mm}$-thick films by Goodfellow, Brazil. Before the plasma processing, the polymeric material were cut into pieces with $20 \times 15 \mathrm{~mm}^{2}$ size. All samples were ultrasonically cleaned twice, first in distilled water and after that in isopropyl alcohol for removing surface contaminants. Finally, the samples were dried for $2 \mathrm{~h}$ in ambient atmosphere. Upon the plasma treatment some polymer samples were rinsed for $1 \mathrm{~min}$ in distilled water, dried for $1 \mathrm{~h}$ in ambient atmosphere and after that subjected to further analysis.

\subsection{Plasma jet}

The APPJ used in this work is basically a one-electrode device, which consists of a standard glass syringe $(10 \mathrm{ml})$, whose inner diameter is $15 \mathrm{~mm}$ with a nozzle inner diameter of $1.0 \mathrm{~mm}$. An insulating cap, through which the working gas can be admitted to the system, closes the syringe end. Argon was introduced into the syringe at controllable flow rate between 0.2 and $2.51 / \mathrm{min}$. A $0.65-\mathrm{mm}$-thick $\mathrm{Ni}-\mathrm{Cr}$ wire is installed on the syringe axis and served as a high voltage electrode. The distance between the tip of the wire electrode and the nozzle entrance was about $2 \mathrm{~mm}$. The device was installed vertically using a dielectric support. A metallic platform $\left(13.0 \times 13.0 \mathrm{~cm}^{2}\right)$, equipped with a moveable stage that can provide controllable displacements in $x-y$ directions, was

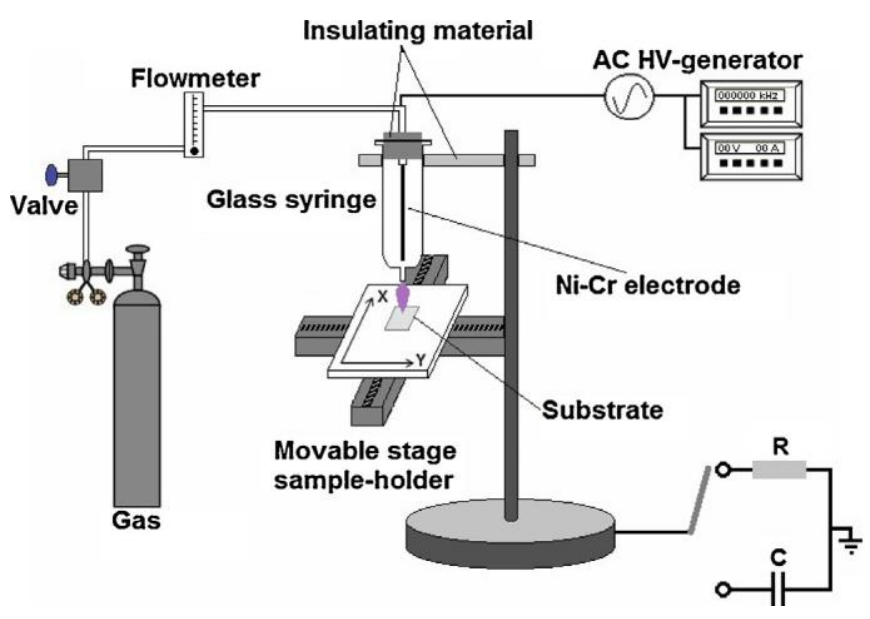

Fig. 1. Schematic diagram of the experimental setup.

situated under the syringe nozzle and acted as a ground electrode. To avoid accidental arcing the whole platform was covered with a 3-mm-thick glass layer (Fig. 1). A Minipuls 4 high voltage power supply (GBS Elektronik GmbH, Dresden, Germany) generates high $\mathrm{AC}$ voltages (up to $24 \mathrm{kV}$ peak-to-peak) within the frequency range of $20-40 \mathrm{kHz}$. A high voltage divider and a serial resistor of $10 \mathrm{Q}$ were used for monitoring the applied voltage and the discharge current. The voltage drop across a serial capacitor of $10 \mathrm{nF}$ is proportional to the transferred charge. The signals were displayed on a digital oscilloscope (Tektronix TDS 2024B, $200 \mathrm{MHz}$ ).

\subsection{Polymer treatments}

The samples to be treated had rectangular shape (dimensions of $2.0 \times 1.5 \mathrm{~cm}$ ) and were placed on the glass that covered the grounded platform. Before starting the plasma treatment each sample was aligned central and perpendicular to the syringe axis at a selected distance from the nozzle exit. Argon gas was admitted into the syringe with a controlled flow rate and consequently the plasma jet was ignited and directed onto the sample. Depending on applied voltage and gas flow rate, the length of the Ar plasma plume can extend up to $2.5 \mathrm{~cm}$. As far as the Ar flow remains in laminar regime $(\operatorname{Re}<2300)$ the plasma plume length scales with the gas flow rate. However for turbulent gas flow the plasma column easily mixture with the ambient air and as a result the jet became instable and its length is reduced [33]. For our experimental conditions (Ar gas and syringed orifice diameter of $1.0 \mathrm{~mm}$ ) the transition between laminar and turbulent regime happens at gas flow around $1.51 / \mathrm{min}$ and above this value the Ar jet length was actually reduced. Therefore to ensure the maximal extension of the plasma plume $(\sim 2.5 \mathrm{~cm})$ for all material processing we used gas flow rate of $1.3 \mathrm{l} / \mathrm{min}$.

Two series of experiments were conducted to evaluate the degree of polymer surface modification induced by the plasma jet. First, at a fixed jet length (maintained $2.5 \mathrm{~cm}$ for all experiments), the sample was static and the distance to the syringe nozzle was varied from 2.0 to $3.5 \mathrm{~cm}$. During the second series of treatments the sample-nozzle distance and the jet extension were kept constant while by using the movable stage the samples were displaced perpendicularly under the plasma jet with a constant velocity of about $6 \mathrm{~mm} / \mathrm{s}$. The scanning route consisted of 4 consecutive parallel paths (along the sample's larger dimension) spaced at $3 \mathrm{~mm}$ distance. Using these scanning parameters an area of approximately $200 \mathrm{~mm}^{2}(17 \mathrm{~mm} \times 12 \mathrm{~mm})$ can be covered for about $15 \mathrm{~s}$. This series of treatments was aimed to achieve a uniform surface modification over the internal part of the sample. 
After establishing the optimal jet operating conditions PE and PP samples were treated by APPJ to assess the process efficiency.

It is well known that in the case of thin films the DBD processing can ensure fast and uniform surface modification over a large area. However for some specific applications involving complex-shaped targets and/or small areas of treatment (as in the microelectronics and some bio and medical applications) the APPJ may turn out to be more attractive as a surface modification tool [23]. Therefore it would be interesting to compare the surface modification efficiency of the APPJ described above with the efficiency of a typical DBD process. In this work for surface modification of the same polymers (PE and PP) we employed the air DBD system described elsewhere [34]. The chemical and morphological modifications of the polymer surface, induced by the APPJ and DBD processes, are compared and discussed in the following section.

\subsection{Diagnostics}

To evaluate the effect of plasma on the polymer surface, the water contact angle was measured by the sessile drop method on a standard Ramé-Hart goniometer, model 300 using the DROPImage software. In the case of plasma jet the dimension of the modified area was investigated in longitudinal scans along the long side of the sample. Sequences of water droplets were deposited on the polymer surface in three parallel lines ( $3 \mathrm{~mm}$ apart). The volume of each water drop was $0.5 \mathrm{fLl}$. The distance between two subsequent drops in each line of measurements was also $3 \mathrm{~mm}$. The longitudinal distributions of WCA were used to obtain an average WCA value, which represented the degree of surface modification of every sample.

The plasma-induced changes in the surface morphology were studied by atomic force microscope (AFM) performed by a Shimadzu SPM9600 Scanning Probe Microscope. During the analysis, the microscope was operated in tapping mode with a scanning rate of $0.5 \mathrm{~Hz}$ for all scanning sizes using etched silicon probe (NSG30 series; $\mathrm{k}=50 \mathrm{~N} / \mathrm{m}$ ). The operating point (potential difference applied between the sample and the cantilever, which in tapping mode determines the oscillation amplitude) was set to $0.172 \mathrm{~V}$. From the AFM topographic profiles on $10.0 \times 10.0 \mathrm{fLm}^{2}$ area the root mean square (rms) roughness of the surface was calculated using the freeware software Gwyddion 2.25.

Photoelectron spectra were recorded on a Kratos XSAM 800 spectrometer operated in fixed analyzer transmission (pass energy $80 \mathrm{eV}$ for wide scans and $40 \mathrm{eV}$ for detailed regions), using $\mathrm{Mg} \mathrm{Ka}_{1,2}$ $(1253.6 \mathrm{eV})$ excitation. The electron take off angle was $90^{\circ}$ that gave depth of information between 10 and $12 \mathrm{~nm}$. The pressure of the analysis chamber was lower than $10^{-7} \mathrm{~Pa}$. The spectra were referenced to the $\mathrm{C} 1 \mathrm{~s}$ line (binding energy, $\mathrm{BE}=285.0 \mathrm{eV}$ ) of the hydrocarbon type carbon. Data acquisition and processing were performed with the Kratos Vision 2 program.

The change in the surface chemical composition of polymers upon plasma exposure was also analyzed by attenuated total reflection infrared spectroscopy ATR-FTIR, using a Perkin Elmer, model Spectrum 100 instrument with diamond as reflection element. The spectra were collected at $4 \mathrm{~cm}^{-1}$ resolution and signal averaging of 8 scans.

\section{Results and discussion}

\subsection{Plasma treatment}

Fig. 2 shows a photograph of the Ar plume taken at a gas flow rate of $1.31 / \mathrm{min}$, a signal frequency of $37 \mathrm{kHz}$ and voltage amplitude of $10 \mathrm{kV}$. The sample was positioned under the plasma jet at a distance of $2.0 \mathrm{~cm}$. Inside the syringe the gas breakdown occurred

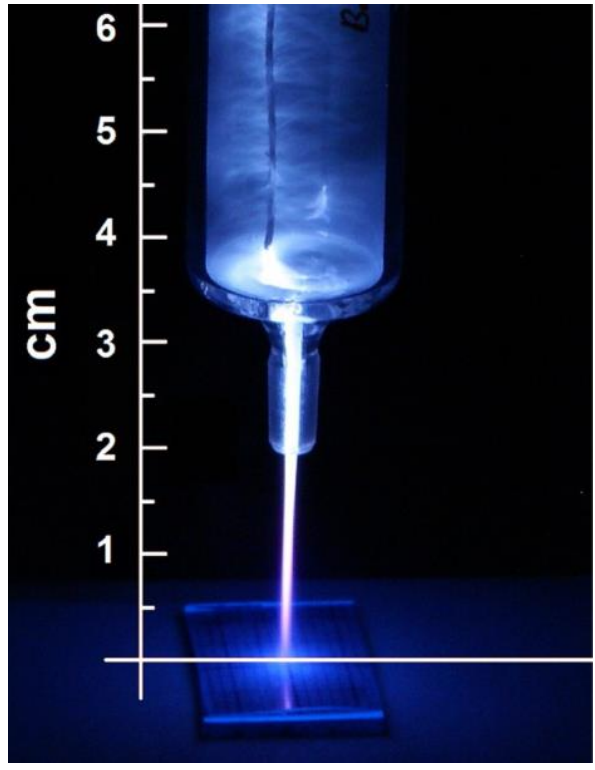

Fig. 2. Photograph of the Ar plasma plume using applied voltage with amplitude of $10 \mathrm{kV}$ and frequency of $37 \mathrm{kHz}$. The Ar gas flow was $1.3 \mathrm{l} / \mathrm{min}$.

in the form of multiple filaments that originated from the powered $\mathrm{Ni}-\mathrm{Cr}$ electrode and terminated to the inner wall of the glass syringe. However, more intense discharge activities always developed between the electrode tip and the syringe nozzle, from where the plasma jet was launched to the air.

The typical waveforms of the applied voltage and the corresponding discharge current have the usual form for an APPJ device and were presented in our previous work [35]. Since the discharge power is consumed in several short current pulses it is not a trivial task to calculate the power dissipated in the discharge. The method commonly employed for obtaining the discharge power is based on the charge-voltage Lissajous figure. The area of the closed Q-V loop for one period of the applied voltage is equal to the energy dissipated per one cycle $[11,15]$. The mean discharge power is then simply the energy per cycle multiplied by the working frequency [27]. A typical Q-V Lissajous figure of the plasma jet is shown in Fig. 3. It was obtained for applied voltage amplitude of $10 \mathrm{kV}, 37 \mathrm{kHz}$ signal frequency, gas flow of $1.31 / \mathrm{min}$ and $2.0 \mathrm{~cm}$ distance between the syringe nozzle and the substrate. At these operating conditions the area of the Lissajous figure shown in Fig. 3 was $120 \mathrm{fLJ}$ giving a power of $4.4 \mathrm{~W}$. The same set of parameters (voltage amplitude of $10 \mathrm{kV}, 37 \mathrm{kHz}$ signal frequency, gas flow of $1.31 / \mathrm{min}$ and $2.0 \mathrm{~cm}$ distance between the syringe nozzle and the substrate) was employed

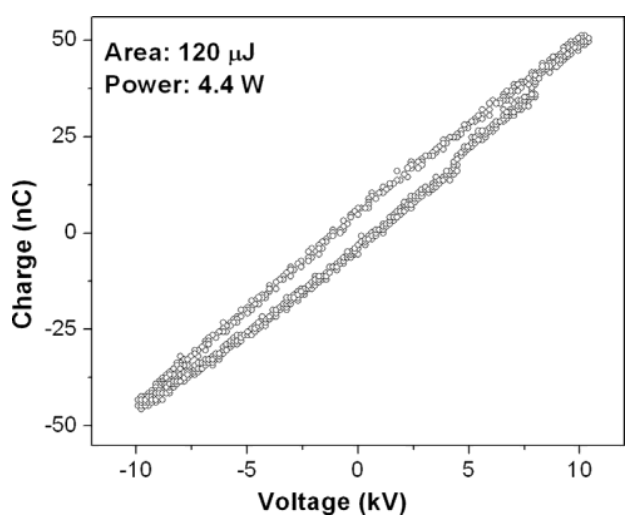

Fig. 3. Lissajous Q-V diagram of APPJ for signal frequency of $37 \mathrm{kHz}$ and sample-tonozzle distance of $2.0 \mathrm{~cm}$. 




Fig. 4. Plot of mean discharge power versus nozzle-substrate distance. The applied voltage has amplitude of $10 \mathrm{kV}$ and frequency of $37 \mathrm{kHz}$. Gas flow rate was $1.3 \mathrm{l} / \mathrm{min}$.

in all subsequent polymer treatments except for only few experiments where effect of the distance between the substrate and the nozzle was investigated. However, in this case, all other jet operation parameters were maintained the same.

Since the moving stage was grounded the device in Fig. 1 can be classified as a kind of DBD-like plasma jet, in which the distance to the sample can significantly influence the device operation. Fig. 4 depicts the plasma jet power for different nozzle-to-substrate distances. Each point in Fig. 4 is the average value of at least 5 measurements. As can be seen for gaps beyond $3.0 \mathrm{~cm}$, the discharge power achieved a saturation value of about $1.5 \mathrm{~W}$ and the jet operated essentially as a one-electrode system. However, at shorter gaps the power increased exponentially due to the capacitive coupling between the grounded platform and the high-voltage electrode. Although the metallic platform was covered with a dielectric barrier, for gaps shorter than $2 \mathrm{~cm}$ the jet power grew very high and the plasma plume became hot and constricted. The excessive heating in this case can cause glass cracking with consequent arcing. Moreover, a very intense plasma jet is not desirable for polymer treatment because it can melt the substrate.

The temperature at the jet effluent was estimated by using a small thermometer. It was inserted in the tip of the plasma plume at approximately $2 \mathrm{~cm}$ from the nozzle and detected temperature under $50{ }^{\circ} \mathrm{C}$. This result demonstrated that at $2.0 \mathrm{~cm}$ distance the $\mathrm{Ar}$ plasma jet can be applied for polymer surface modification without thermal damage to the surface.

In the next experiment we applied the Ar plasma jet for surface modification of polyethylene terephthalate (PET) and studied the degree of polymer surface modification as well as its spatial distribution. PET samples $(20 \mathrm{~mm} \times 15 \mathrm{~mm})$ were centered under the syringe nozzle and treated with APPJ at different nozzle-platform distances. The treatment time was set to $30 \mathrm{~s}$ and the voltage magnitude and frequency as well as the gas flow rate was fixed at $10 \mathrm{kV}$, $37 \mathrm{kHz}$ and $1.3 \mathrm{l} / \mathrm{min}$, respectively.

\subsection{Polymer wettability}

The main processes responsible for surface modification of polymers by atmospheric plasmas are hydrogen abstraction, surface etching and incorporation of polar groups [14-16]. Cross-linking of plasma-created radials on the polymer surface is also possible. The chemical changes as well as the surface roughening lead to change of material wetting characteristics, which can be detected by water contact angle measurements [17].

Fig. 5 shows the water contact angle (WCA) as a function of the water drop position on the PET sample treated by APPJ. The untreated PEThas WCA of $78^{\circ}$. Each point in Fig. 5 is the mean value of three WCA measurements at the same longitudinal position

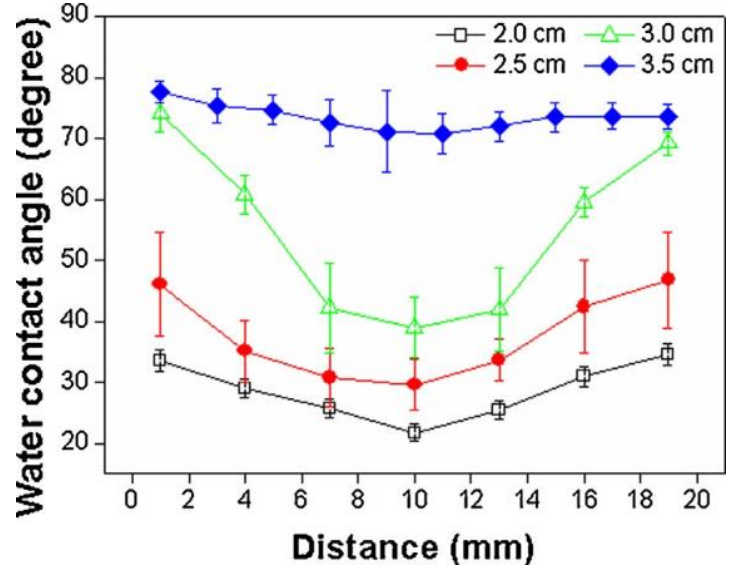

Fig. 5. Longitudinal WCA profiles of PET samples exposed to Ar plasma jet for different distances. The static samples were centered under the plasma jet and treated for $30 \mathrm{~s}$. A constant gas flow of $1.3 \mathrm{l} / \mathrm{min}$ and signal amplitude and frequency of $10 \mathrm{kV}$ and $37 \mathrm{kHz}$ were used.

but $3 \mathrm{~mm}$ apart along the short sample side. One can see that the polymer wettability was increased mostly in the center of sample (at about $10 \mathrm{~cm}$ from the sample edge), where was the position of the plasma jet effluent. At $3.5 \mathrm{~cm}$ distance between the substrate and the nozzle the polymer surface modification is insignificant because the active species recombined before reaching the PET surface. However at $3.0 \mathrm{~cm}$ gap the WCA in the central part of the sample was reduced substantially. Since the plasma plume visually did not touch the substrate a possible modification agent is the VUV/UV radiation produced by the Ar discharge. Since the UV photons propagate preferentially along the plasma column they would rather interact only with the target surface that is located just under the plasma jet [33]. As shown in [36] besides the VUV/UV radiation the long-lived active species, like $\mathrm{O}_{3}$ can also reach the surface and spread radially over a large area of the sample. As can be noted in Fig. 5 the characteristic width (FWHM) of the WCA longitudinal distribution is much bigger than the diameter of the Ar plasma column. Therefore one can conclude that in this case the ozone produced by the jet-air interaction is the major modification agent responsible for the enhanced surface hydrophilicity.

The WCA values become increasingly smaller when the treatment distance is further reduced. Also, at distances shorter than $3.0 \mathrm{~cm}$ the surface modification spanned over much larger area. Two effects can explain these findings. First, as can be seen in the Fig. 4 for distances less than $3.0 \mathrm{~cm}$ the jet power increased significantly resulting in production of more active species. Second, at these operating conditions the Ar plasma plume $(2.5 \mathrm{~cm}$ long) actually touched the surface forming a wide spot (Fig. 2). In this case, the gas flow reflected from the surface and dispersed radially outward thus spreading the plasma and the active species over larger area of the sample [17]. Now not only the ozone but also some short-living active species, such as, atomic oxygen and $\mathrm{OH}$ radical may interact with the central part of the substrate leading to an enhanced hydrophilicity. As shown in $[14,36]$ the atomic oxygen can actually spread up to $2.0-2.5 \mathrm{~mm}$ way from the plasma column. The computer modeling of active species generated by $\mathrm{Ar}$ plasma jet in [31] predicted that along the gas flow oxygen atoms could cover even larger distances $(\sim \mathrm{cm})$. Of course as a long-living species, the ozone will spread over even larger area of the sample. Therefore for jet-to-sample distances of 2.0 and $2.5 \mathrm{~cm}$ the modified area of the polymer surface was much bigger than the APPJ cross-section [32,33].

For the next set of treatments, the distance to the platform was fixed and using the movable stage the sample was displaced under the jet. For the sample scanning conditions described in the Section 


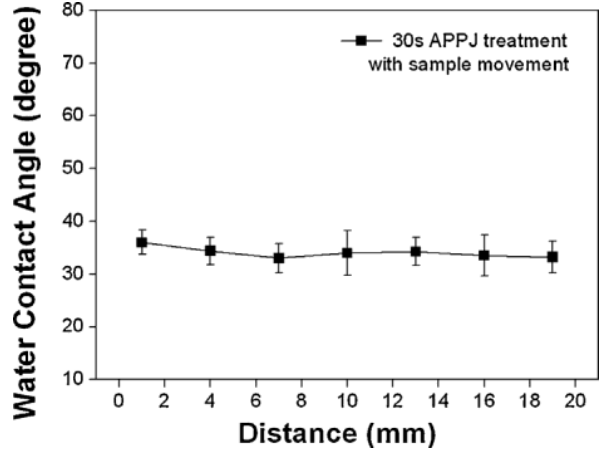

Fig. 6. Effect of the sample reciprocation on the PET wettability. The samples were placed $2.0 \mathrm{~cm}$ under the nozzle and treated for $30 \mathrm{~s}$ ( 2 scans). The treatment parameters were the same as in Figure 5.

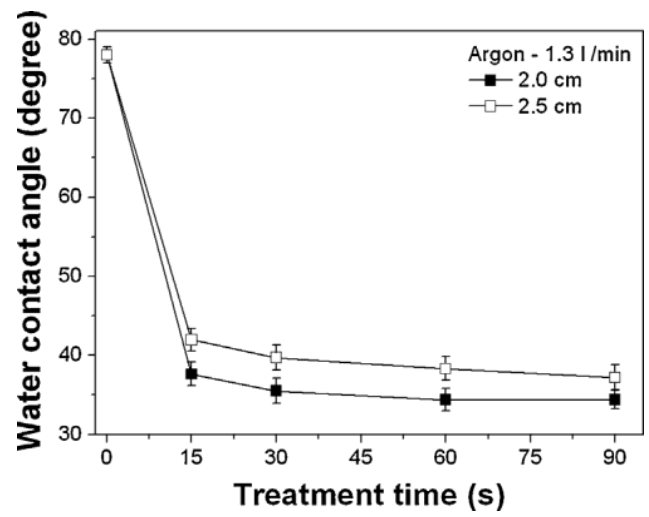

Fig. 7. Variation of PET water contact angle with APPJ treatment time for two different distances to the substrate.

2.3 an area of approximately $200 \mathrm{~mm}^{2}$ can be covered for about $15 \mathrm{~s}$. The APPJ treatments with sample reciprocation were conducted for $15,30,60$ and $90 \mathrm{~s}$, which corresponded to $1,2,4$ and 6 scans of the selected sample area. The mean value of WCA along the long side of the PET sample is presented in Fig. 6. The values of WCA in the Fig. 6 are quite close, except for a slightly bigger deviation in the left side of sample, which is probably caused by a small sample misalignment. As can be seen when the sample was moved during the APPJ treatment only two scans were sufficient to provide quite uniform PET surface modification over the selected area of the sample. From the WCA longitudinal distribution in Fig. 6 an average angle of about $35^{\circ}$ was calculated. This value is considered as the contact angle of the polymer surface. For all subsequent treatments of polymers (conducted at the above describe sample scanning conditions) the average WCA value was calculated by the same procedure and used for further comparison and analysis.

In Fig. 7 is shown the average WCA of PET samples as a function of the treatment time (or the surface scans) using two different distances from the syringe nozzle. Samples treated at the shorter distance exhibit slightly smaller WCAs because larger amount of reactive radicals can reach the surface and interact with it. The polymer wettability tends to increase with the time of plasma exposure. However, after four scans of the surface (or $60 \mathrm{~s}$ plasma exposure) the WCA achieved a steady value, which did not further decreased with the time of treatment. This kind of behavior is typical for the plasma-treated polymers and it is caused by the saturation of grafting sites on the polymer surface. Beyond some critical time of treatment the polymer surface exhibits more or less stable WCAs. Therefore, one way to assess the efficiency of different plasma processes is to compare the steady WCA of polymer surface that was obtained as a result of the treatments. As it will be shown below
Table 1

Water contact angles of PE and PP polymers treated by APPJ or DBD.

\begin{tabular}{lll}
\hline Polymer & Treatment & WCA (deg) \\
\hline PE & None & $93.7 \pm 1.2$ \\
& DBD & $49.0 \pm 2.3$ \\
& APPJ & $36.2 \pm 2.5$ \\
PP & None & $102.3 \pm 2.3$ \\
& DBD & $63.1 \pm 3.0$ \\
& APPJ & $52.0 \pm 2.6$ \\
\hline
\end{tabular}

the steady WCAs of polymer surfaces depend also on what kind of plasma source (APPJ or DBD) was used for the treatment.

To test further the efficiency of APPJ in material surface modification, experiments were conducted with polyethylene PE and polypropylene PP. The plasma jet was operated at the same conditions $-10 \mathrm{kV}, 37 \mathrm{kHz}$, gas flow rate of $1.3 \mathrm{l} / \mathrm{min}$ and the distance to the sample was set to $2.0 \mathrm{~cm}$. The polymer substrates were reciprocated at the same conditions of scanning as the PET samples in the preliminary study using treatment time of $60 \mathrm{~s}$. As evidenced by the Fig. 7 his treatment time is more than enough to saturate the polymer wetting properties. For comparison the same polymers (PE and PP) were treated by the DBD system described in [34]. As it was shown there, the DBD processing of polymers is also characterized by a critical treatment time (10-20 s) beyond which there is no further reduction of WCA. Therefore to make sure that the WCA of the polymer surface achieved its steady value the treatment time was set to $30 \mathrm{~s}$. The other DBD treatment parameters were: peak voltage of $12 \mathrm{kV}$, signal frequency of $20 \mathrm{kHz}$, inter-electrode gap of $3.0 \mathrm{~mm}$ and power per unit area of about $1.0 \mathrm{~W} / \mathrm{cm}^{2}$. The WCA values of PE and PP polymers for both treatments are shown in the Table 1.

It can be noticed that the polymer wettability was improved by the APPJ treatment in higher extend as by the DBD treatment. The wettability is strongly influenced by the surface chemical composition. As it will be shown by the XPS analysis presented below, in the case of APPJ processing more $\mathrm{O}$ atoms were attached to the polymer chain, which led to an enhancement of the surface hydrophilicity.

\subsection{Surface chemical composition}

The surface chemical composition of the plasma-treated polymers was evaluated by XPS analysis. The elemental composition of PE and PP polymers treated by DBD and APPJ is shown in Table 2. Uncertainty in at $\%$ values presented there depends on intensity of signal - for strong signals is $\sim 10 \%$, for weak signals it can go up to $30 \%$. Therefore the last digit in the atomic concentration values and the $\mathrm{O} / \mathrm{C}$ ratios shown in Table 2 is not meaningful and it is only shown to indicate a tendency.

The stability of a modified surface is a serious issue in the field of material surface modification. Usually, the plasma-treated polymers exhibit so-called hydrophobic recovery. It is manifested by a partial recovery of the original material wetting properties upon

Table 2

Atomic concentrations obtained from XPS survey spectra of PP and PE films exposed to APPJ and DBD.

\begin{tabular}{llrlll}
\hline Polymer & Treatment & $\mathrm{O}$ at $\%$ & $\mathrm{C}$ at $\%$ & $\mathrm{~N}$ at $\%$ & $\mathrm{O} / \mathrm{C}$ \\
\hline PE & None & 1.5 & 98.5 & & 0.02 \\
& DBD & 15.0 & 83.5 & 1.5 & 0.18 \\
& DBD-washed & 7.0 & 92.0 & 1.0 & 0.08 \\
& APPJ & 27.0 & 73.0 & & 0.37 \\
& APPJ-washed & 19.5 & 76.5 & & 0.25 \\
PP & 2.0 & 98.0 & & 0.02 \\
& None & 13.5 & 84.5 & 2.0 & 0.16 \\
& DBD & 5.5 & 92.5 & 1.0 & 0.06 \\
& DBD-washed & 27.0 & 73.0 & & 0.37 \\
& APPJ & 85.5 & & 0.15
\end{tabular}


rinsing it with water or other polar solvent. As inferred in previous works $[21,34]$ this behavior is caused by the dissolution of low molecular weight oxidized material (LMWOM). It is constituted of short highly oxidized polymer fragments, loosely bounded to the surface, which were produced during the plasma exposure. The loss of this material due to dissolution in polar solvent or evaporation results in reduction of surface wettability. To investigate this effect prior to XPS analysis some plasma-treated polymer samples were rinsed in distilled water.

The pristine PE and PP samples exhibit small amounts of oxygen (several at\%) due to surface contamination. After the treatments the polymers became more oxidized and the $\mathrm{O} / \mathrm{C}$ ratio increased significantly, which is in agreement with the enhanced wettability of the samples.

Both treatments, the DBD and APPJ, added $\mathrm{O}$ atoms to the polymer surfaces, however some differences can be noticed. First of all, it is clear, that the APPJ processing led to more intense surface oxidation of all polymer samples. This is in an agreement with the WCA measurements, which showed that polymers exposed to APPJ exhibit higher hydrophilicity. Moreover, even after rinsing the samples in water the polymers treated by APPJ still exhibit much higher oxygen content than the ones treated with DBD. On the other hand, the DBD process also attached to the surface small amount of $\mathrm{N}$ atoms, which did not happen in the case of plasma jet treatment. This finding can be explained by the fact that the gas flow in plasma jet carries the active species produced by interaction of Ar plasma column with the air to the sample surface where they react with the polymer chain. Computer simulations in [31] showed the O-related active species produced by the interaction of Ar plasma jet with humid air could propagate downstream the jet up to $2.5-3.0 \mathrm{~cm}$. On the other hand the excited and ionized nitrogen species have short lifetime and cannot reach the surface in the case of the APPJ treatment. Of course in a DBD process, where the plasma is in a direct contact with the surface some $\mathrm{N}$ atoms can be also attached to the surface.

The decomposition of $\mathrm{C} 1 \mathrm{~s}$ peak can be used to gain insight into the nature of polymer surface modification caused by the plasma. As an example the C1s peaks of two PP samples are presented in Figs. 8( $\mathrm{a}$ and $\mathrm{b})$. Since the $\mathrm{C} 1 \mathrm{~s}$ peaks of the PE samples present the same features they are not shown here. The pristine polymers are characterized by quite narrow $\mathrm{C} 1 \mathrm{~s}$ peaks. They were decomposed into two peaks: $\mathrm{C} 1 \mathrm{C}-\mathrm{H}$ and $\mathrm{C}-\mathrm{C}$ bonds) at $285.0 \mathrm{eV}$ and a small component $\mathrm{C} 2$ (C-O bonds) at $286.5 \mathrm{eV}$ due to slight surface contamination. However, after the polymer treatment a broad shoulder emerged at the high binding energy side of the C1s peak indicating oxygen incorporation. The spectra of plasma-treated polymers were deconvoluted into four components as follows: $\mathrm{C} 1$ - hydrocarbons $\mathrm{C}-\mathrm{H}$ or $\mathrm{C}-\mathrm{C}$ at $285.0 \mathrm{eV}$; $\mathrm{C} 2$ - carbon atoms singly bounded to oxygen ( $\mathrm{C}-\mathrm{O}$ at $286.5 \mathrm{eV}) ; \mathrm{C} 3$ - carbon atoms with single or double bonds to oxygen atoms $(\mathrm{O}-\mathrm{G}-\mathrm{O}$ or $\mathrm{E}-\mathrm{O}$ at $288.0 \mathrm{eV})$ and $\mathrm{C} 4-$ carboxyl group- $\mathrm{COOH}$ at $289.0 \mathrm{eV}$. Details of surface chemical composition of plasma-treated PP and PE polymers are presented in the form of bar-plots in Fig. 9(a) and 9(b), respectively. It can be noted that the APPJ process produced higher amount of triply oxidized carbon species $(\mathrm{O}-\mathrm{C}=\mathrm{O}$ group) than the DBD treatment, suggesting more intense surface oxidation. In fact, the plasma chemistry in DBD and APPJ processes is quite different. In DBD the active species are mostly produced by collisions with energetic electrons while in the APPJ the O species are produced by interaction of Ar metastables with the air molecules. Apparently, the plasma jets are very efficient in producing long-living oxygen species [31,32] that being carried by the gas flow can reach the surface and interact with it.

For the samples treated by APPJ and DBD, rinsing with water led to a reduction of the oxygen content on the surface. As a rule the most oxidized carbon components (C4 and $\mathrm{C} 3$ ) are removed from the surface (Fig. 9). This is consistent with the dissolution of


Fig. 8. Curve-fitted C $1 \mathrm{~s}$ peaks of (a) pristine PP and (b) PP treated by Ar plasma jet.
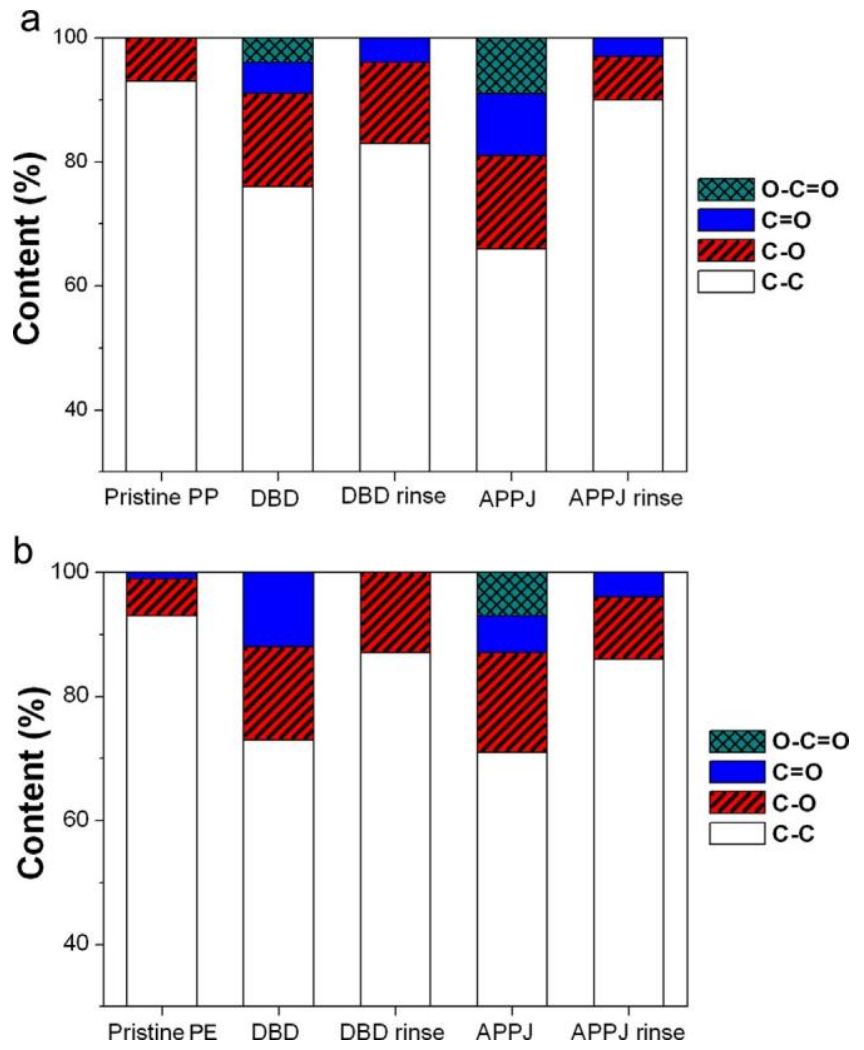

Fig. 9. Bar-plot presenting surface chemical composition of (a) PP and (b) PE polymers obtained from the decomposition of XPS C1s peaks. 
some LMWOM formed on the surface during the plasma processing. However, the washed PP and PE surfaces still posses more $\mathrm{O}$ atoms than the pristine samples, which indicates that the oxygen is not only localized in dissoluble LMWOM, but some $\mathrm{O}$ atoms are bounded to the polymer chain. Also, as indicated by the XPS elemental analysis, in the case of APPJ treatment the amount of $\mathrm{O}$ atoms left on the surface of the washed PE and PP samples is more than twice higher compared to the $\mathrm{O}$ on the samples treated with DBD.

When polymers are treated by atmospheric plasma an amorphous layer, composed by LMWOM, some impurities and adsorbed gas molecules, is formed on the surface. Therefore, to bind to the polymer oxygen atoms should first get through this outmost surface layer. So, only a part of the active species that approached the surface can be actually attached to the polymer chain. Most of the oxygen moieties are concentrated in the LMWOM, which is formed on the surface during the treatment. However, in the case of APPJ treatment two effects probably help to add more oxygen atoms on the surface. First, the gas flow from the jet carries the active species close to the surface helping them penetrate through the interface layer. Also, the gas stream may partly remove the viscous LMWOM from the surface uncovering the polymer chain below, where more $\mathrm{O}$ atoms can be attached.

The results from XPS analysis are further corroborated by ATRFTIR analysis. The plasma treatments at atmospheric pressure affect only the utmost layer of the surface that is way it is not an easy task to observe surface chemical modification by FTIR analysis. However, in the case of heavy surface oxidation of polymers their infrared spectra may present some new characteristics.

Fig. 10(a) and (b) and show the Fourier transformed infrared spectra of polyethylene and polypropylene before and after plasma treatment, respectively. The polymers were treated using the same plasma conditions described in experimental methods with 4 complete scans of the sample surface. The spectra of pristine PE and PP basically present only the characteristic vibration modes of $-\mathrm{CH}_{2}-$ and $-\mathrm{CH}_{3}-$ groups. Exposure of polymers to APPJ causes the appearance of several new features in the infrared spectra. The broad band between 3100 and $3600 \mathrm{~cm}^{-1}$ is due to the O_H stretch of alcohols. The plasma-treated PE and PP polymers exhibit bands around $1640 \mathrm{~cm}^{-1}$ and $1730 \mathrm{~cm}^{-1}$ that are related to the $\in \mathrm{O}$ bond. The band at $1640 \mathrm{~cm}^{-1}$ is assigned to $C \mathrm{O}$ stretch of hydrogen bonded carboxylic acid group. Furthermore, the band at $1730 \mathrm{~cm}^{-1}$ is associated to $\mathrm{C}=\mathrm{O}$ stretch of ketone. In addition, the spectrum of $\mathrm{PE}$ exhibits a band around $1150 \mathrm{~cm}^{-1}$ that can be attributed to $\mathrm{O}-\mathrm{C}$ stretch in $\mathrm{C}-\mathrm{OH}$ groups [37].

\subsection{Surface morphology}

For plasma processing at atmospheric pressure the sputtering is negligible and the changes of surface morphology are mainly due to etching by reactive species. In the case of plasma jets, the exited and ionized argon atoms from the discharge create reactive species (mostly atomic oxygen, ozone and $\mathrm{OH}$ radical $[32,36]$ ) by reaction with the ambient air. These highly reactive species can etch polymers and roughen their surface $[14,36]$. The AFM images of the untreated PP and the polymer samples treated by APPJ at different processing time are presented in Fig. 11. Considerable topographical changes due to the plasma exposure can be observed. Kind of grain-like structures, whose size tends to increase with the time of treatment, are formed on the polymer surfaces. AFM images of plasma-treated PE samples (not shown here) exhibit very similar characteristics. The nodules on intrinsically hydrophobic polymer surfaces can be explained by the formation of polar LMWOM, which agglomerates into spherically shaped structures. Upon washing the samples in distilled water the large structures disappear, which confirms that, they were, in fact, constituted by
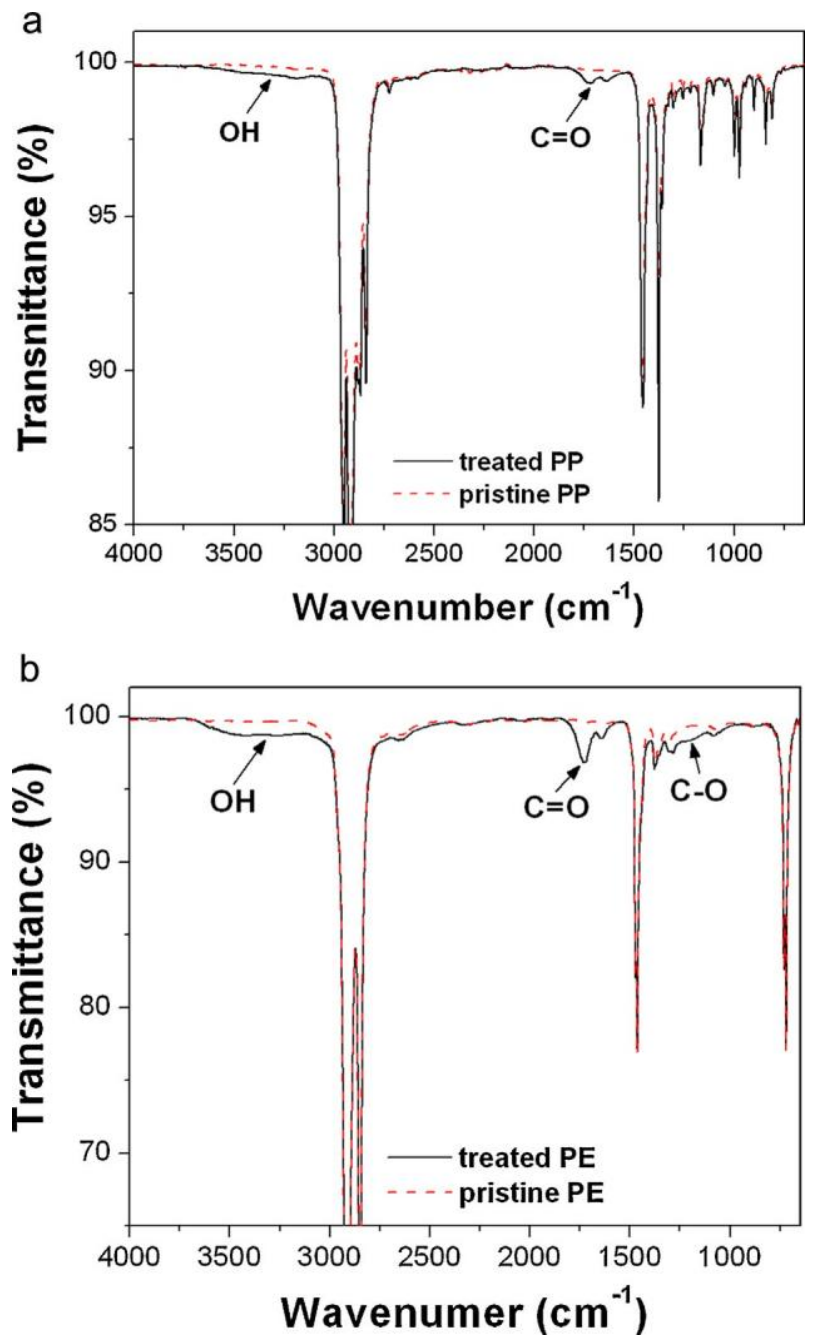

Fig. 10. Infrared spectra of (a) PE and (b) PP before and after plasma treatment.

a soluble LMWOM. This finding is in an agreement with the XPS analysis that indicated a reduction of the surface oxygen content upon rinsing. The washed surfaces exhibit only small-size irregularities that are result from surface etching [38]. Table 3 shows the roughness of PE and PP after the plasma treatment alone and in the case of polymer treatment followed by sample washing. Basically, the polymer roughness tends to increase with increasing the treatment time, which is related to the prolonged surface etching effect. Upon washing the polymer roughness slightly increases in comparison to the plasma-treated samples. Rinsing basically removes from the surface short polymer fragments and amorphous phases that were agglomerated in large and smooth structures on the surface in that way uncovering many protrusions and deep craters on the surface.

Table 3

Polymer roughness before and after the treatment with APPJ. Some samples were rinsed after the plasma exposure.

Time of APPJ RMS roughness (nm)

\begin{tabular}{|c|c|c|c|c|}
\hline \multirow{2}{*}{ treatment (s) } & \\
\hline & PE & PE washed & PP & PP washed \\
\hline 0 & $8.5 \pm 0.4$ & $8.5 \pm 0.4$ & $5.1 \pm 0.3$ & $5.1 \pm 0.3$ \\
\hline 15 & $41.6 \pm 1.5$ & $30.9 \pm 1.3$ & $18.3 \pm 0.7$ & $13.9 \pm 1.3$ \\
\hline 30 & $39.6 \pm 3.3$ & $42.9 \pm 3.7$ & $21.1 \pm 2.0$ & $28.3 \pm 4.0$ \\
\hline 60 & $57.8 \pm 5.3$ & $61.2 \pm 6.1$ & $62.1 \pm 7.0$ & $66.4 \pm 5.0$ \\
\hline
\end{tabular}





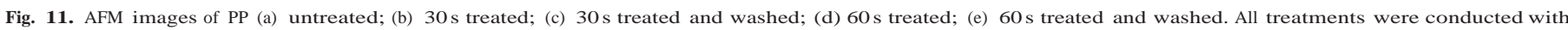
sample reciprocations using signal amplitude $10 \mathrm{kV}$, frequency of $37 \mathrm{kHz}$ and Ar flow of $1.3 \mathrm{l} / \mathrm{min}$.

Comparing the morphology of the samples treated by APPJ with the one of polymers treated by DBD in our previous work [34] it appears that both processes produce LMWOM. However, it seems that the DBD-treated polymers exhibit much larger nodulelike structures on their surfaces. This enhanced accumulation of oxidized material on the surface is probably due to the more intense polymer degradation during the DBD processing. In this case, besides the attack of oxygen-related active species, the surface also is subjected to charged particles and energetic UV photons that can cause extensive breakage of the polymer chain. On the other hand in the APPJs treatments the effect of UV-photons and charge particles on the surface is greatly reduced. Therefore the surface structural modification in APPJ treatments is mostly caused by the active oxygen related species that are brought to the surface by the gas stream. The latter may also disperse radially outward the LMWOM formed on the surface, so that the $\mathrm{O}$ atoms produced in the plasma plume, can reach and effectively etch and/or attach to the polymer chain.

\section{Conclusion}

The wettability of PE, PP and PET polymers has been enhanced using an atmospheric pressure argon jet in air. If the process conditions, such as, jet-to-sample distance, kind of treatment (static substrate or process with sample scanning) and the time of plasma exposure are properly chosen the APPJ can uniformly modified a selected area of the sample. For comparison, the same polymers were treated with a conventional air DBD system. The XPS and FTIR analysis reveal that both processes led to formation of alcohols, carbonyls and carboxylic acids on the surface of each polymer sample. Furthermore, the plasma exposure results in alteration of the surface morphology mostly through formation of polar LMWOM, which agglomerates into nodule-like structures. Upon rinsing the samples in water the soluble LMWOM was removed from the surface resulting in decrease of oxygen content. In all cases the polymers processed by APPJ exhibited higher oxygen content and better hydrophilicity that the ones treated by DBD. This finding can be attributed to two effects: First is the very efficient chemistry of the Ar plasma jet, where the interaction of Ar metastables with air molecules results in the production of a large amount of reactive species. Secondly, the gas stream of the plasma jet carries the active oxygen species near the surface. Also the stream may partly displace the LMWOM formed on the surface thus uncovering the polymer chain below. The results from this work suggest that atmospheric pressure plasma jet is an efficient tool in polymer surface modification, which can be useful in some applications that require small area of treatment.

\section{References}

[1] S. Samukawa, M. Hori, S. Rauf, K. Tachibana, P. Bruggeman, G. Kroesen, J.C. Whitehead, A.B. Murphy, A.F. Gutsol, S. Starikovskaia, U. Kortshagen, J. Boeuf, T.J. Sommerer, M.J. Kushner, U. Czarnetzki, N. Mason, The 2012 plasma roadmap, J. Phys. D: Appl. Phys. 45 (2012) 253001.

[2] M. Strobel, V. Jones, C.S. Lyons, M. Ulsh, M. Kushner, R. Dorai, M.C. Branch, A comparison of corona-treated and flame-treated polypropylene films, Plasma Polym. 8 (2003) 61.

[3] V.I. Gibalov, G.J. Pietsch, Dynamics of dielectric barrier discharges in different arrangements, Plasma Sources Sci. Technol. 21 (2012) 024010.

[4] X. Lu, M. Laroussi, V. Puech, On atmospheric-pressure non-equilibrium plasma jets and plasma bullets, Plasma Sources Sci. Technol. 21 (2012) 034005.

[5] X. Lu, Z. Jiang, Q. Xiong, Z. Tang, Y. Pan, A single electrode room-temperature plasma jet device for biomedical applications, Appl. Phys. Lett. 92 (2008) 151504.

[6] J.L. Walsh, J.J. Shi, M.G. Kong, Contrasting characteristics of pulsed and sinusoidal cold atmospheric plasma jets, Appl. Phys. Lett. 88 (2006) 171501.

[7] G. Wei, C. Ren, M. Qian, Q. Nie, Optical and electrical diagnostics of cold Ar atmospheric pressure plasma jet generated with a simple DBD configuration, IEEE Trans Plasma Sci. 39 (2011) 1842.

[8] J. Zhang, J. Sun, D. Wang, X. Wang, A novel cold plasma jet generated by atmospheric dielectric barrier capillary discharge, Thin Solid Films 506-507 (2006) 404.

[9] Y. Xian, X. Lu, S. Wu, P.K. Chu, Y. Pan, Are all atmospheric pressure cold plasma jets electrically driven? Appl. Phys. Lett. 100 (2012) 123702.

[10] Q. Nie, A. Yang, Z. Wang, H. Li, X. Zhang, C. Bao, Characteristics of atmospheric room-temperature argon plasma streams produced using a dielectric barrier discharge generator with a cylindrical screwlike inner electrode, IEEE Trans. Plasma Sci. 40 (2012) 2172. 
[11] A. Sarani, A.Y. Nikiforov, C. Leys, Atmospheric pressure plasma jet in Ar and $\mathrm{Ar} / \mathrm{HO}_{2}$ mixtures: Optical emission spectroscopy and temperature measurements, Phys. Plasmas 17 (2010) 063504.

[12] S. Hofmann, A.F.H. van Gessel, T. Verreycken, P. Bruggeman, Power dissipation, gas temperatures and electron densities of cold atmospheric pressure helium and argon RF plasma jets, Plasma Source Sci. Technol. 20 (2011) 065010.

[13] Y. Hong, N. Lu, J. Pan, J. Li, Y. Wu, K.F. Shang, Characteristic study of cold atmospheric argon jets with rod-tube/rode high voltage electrode, J. Eletrostatics 71 (2013) 93.

[14] K. Fricke, S. Reuter, D. Schroeder, V.S. von Gathen, K. Weltmann, T. Woedtke, Investigation of surface etching of Poly(Ether Ether Keton) by atmosphericpressure plasmas, IEEE Trans. Plasma Sci. 40 (2012) 2900.

[15] X. Shao, G. Zhang, J. Zhan, G. Xu, Research on surface modification of polytetrafluoroethylene coupled with argon dielectric barrier discharge plasma jet characteristics, IEEE Trans. Plasma Sci. 39 (Part 2) (2011) 3095.

[16] U. Lommatzsch, D. Pasedag, A. Baalmann, G. Ellinghorst, H. Wagner, Atmospheric pressure plasma jet treatment of polyethylene surface for adhesion improvement, Plasma Process. Polym. 4 (2007) S1041.

[17] E.J. Szili, S.A. Al-Bataineh, P.M. Bryant, R.D. Short, J.W. Bradley, D.A. Steele, Controlling the spatial distribution of polymer surface treatment using atmospheric pr-pressure microplasma jets, Plasma Process. Polym. 8 (2011) 38.

[18] Z. Cao, Q. Nie, D.L. Bayliss, J.L. Walsh, C.S. Ren, D.Z. Wang, M.G. Kong, Spatially extended atmospheric plasma arrays, Plasma Sources Sci. Technol. 19 (2010) 025003.

[19] Y. Song, D. Liu, W. Wang, L. Ji, Q. Zhang, W. Ni, J. Niu, The brush-shaped device used to generate atmospheric and homogeneous plasmas for biomedical applications, Plasma Process. Polym. 10 (2013) 88

[20] E.R. Ionita, M.D. Ionita, E.C. Stancu, M. Teodorescu, G. Dinescu, Small size plasma tool for material processing at atmospheric pressure, Appl. Surf. Sci. 255 (2009) 5448

[21] K. Gotoh, A. Yasukawa, K. Taniguchi, Water contact angle on Poly(Ethylene Terephtalate) film exposed to atmospheric pressure plasma, J. Adhesion Sci. Technol. 25 (2011) 307.

[22] H. Ayan, E.D. Yildirim, D.D. Pappas, W. Sun, Development of a cold atmospheric pressure microplasma jet for freeform cell printing, Appl. Phys. Lett. 99 (2011) 111502.

[23] W. Yan, ZJ. Han, W.Z. Liu, X.P. Lu, B.T. Phung, K. Ostrikov, Designing atmospheric-pressure plasma sources for surface engineering of nanomaterials, Plasma Chem. Plasma Process 33 (2013) 479.
[24] S. Tajima, S. Tsuchiya, M. Matsumori, S. Nakatsuka, T. Ichiki, High-rate reduction of cupper oxide using atmospheric-pressure inductively coupled plasma microjets, Thin Solid Films 519 (2011) 6773.

[25] J.L. Walsh, F. Iza, N.B. Janson, V.J. Law, M.G. Kong, Three distinct modes in an atmospheric pressure plasma jet, J. Phys. D: Appl. Phys. 43 (2010) 075210.

[26] E. Karakas, M.A. Akmar, M. Laroussi, The evolution of atmospheric-pressure low-temperature plasma jets: jet current measurements, Plasma Sources Sci. Technol. 21 (2012) 034016.

[27] Z. Hao, S. Ji, A. Qiu, Study on the influence of the dielectric barrier materials on the characteristics of atmospheric plasma jet in ar, IEEE Trans. Plasma Sci. 40 (2012) 2822.

[28] G. Cho, H. Lim, J. Kim, D.J. Jin, G.C. Kwon, E. Choi, H.S. Uhm, Cold plasma jets made of a syringe needle coved with a glass tube, IEEE Trans. Plasma Sci. 39 (2011) 1234

[29] M.H. Chiang, K.C. Liao, I.M. Lin, C.C. Lu, H.Y. Huang, C.L. Kuo, J.S. Wu, Modification of hydrophilic properties of a polypropylene films by a parallel-plate nitrogenbased dielectric barrier discharge jet, IEEE Trans. Plasma Sci. 38 (2010) 1489

[30] A. Sarani, N. De Geyter, A.Y. Nikiforov, R. Morrent, C. Leys, J. Hubert, F. Reniers, Surface modification of PTFE using an atmospheric pressure plasma jet in argon and argon $+\mathrm{CO}_{2}$, Surf. Coat. Technol. 206 (2012) 2226

[31] W. Van Gaens, A. Bugaerts, Kinetic modeling for an atmospheric pressure argon plasma jet in humid air, J. Appl. Phys. D: Appl. Phys. 46 (2013) 275201.

[32] X. Lu, S. Wu, On the active species concentration of atmospheric pressure nonequilibrium plasma jets, IEEE Tran. Plasma Sci. 41 (2013) 2313.

[33] O.T. Olabanji, J.W. Bradley, Side-on surface modification of Polystyrene with atmospheric pressure microplasma jet, Plasma Process. Polym. 9 (2012) 929.

[34] K.G. Kostov, T.M.C. Nishime, L.R.O. Hein, A. Toth, Study of polypropylene surface modification by air dielectric barrier discharge operated at two different frequencies, Surf. Coat. Technol. 234 (2013) 60.

[35] C.B. Mello, K.G. Kostov, M. Machida, L.R. De Oliveira Hein, K.A. De Campos, Surface modification of polycarbonate by atmospheric-pressure plasma jets, IEEE Trans. Plasma Sci. 40 (2012) 2800.

[36] D. Schroeder, H. Bahre, N. Knake, J. Winter, T. los Acros, V.S. der Garthen, Influence of target surface on the atomic oxygen distribution in the effluent of a micro-scaled atmospheric pressure plasma jet, Plasma Sources Sci.Technol. 21 (2012) 024007.

[37] E. Gonzales II, R.F. Hicks, Surface analysis of polymers treated by remote atmospheric pressure plasma, Langmuir 26 (2010) 3710

[38] K. Gotoh, Y. Kobayashi, A. Yasukawa, Y. Ishigami, Surface modification of PET films by atmospheric pressure plasma exposure with three reactive gas sources, Colloid Polym. Sci. 290 (2012) 1005 